\section{Basic Science of Nuclear Medicine: The Bare Bone Essentials}

\author{
K. H. Lee
}

Reston, VA: Society of Nuclear Medicine and Molecular Imaging, 2015, 374 pages, $\$ 96$

Basic Science of Nuclear Medicine: The Bare Bone Essentials is a concise manual on nuclear physics, instrumentation, and radiation safety. This paperback publication contains 15 chapters and is printed in color. The colored pictorial representations of nuclear medicine technology concepts and images is what makes this publication so unique. This book would be a great complement to a student's board prep materials, a wonderful refresher for the seasoned technologist, and a phenomenal exhibit for the inquisitive patient.

The author, Lee, completes the book with a glossary, bibliography, index, and preface. In the preface, Lee describes the book as being a compilation of important points that may be obscured in more comprehensive textbooks. I would agree. Lee also operates on the assumption that many readers have an aversion to math, and therefore only necessary mathematics have been included. Concepts in the book are repeatedly supported with creative graphics, which are superb in helping the reader visualize the concepts and are a strength of the publication.

The 15 chapters are logically organized by beginning with foundational radiation physics and working through instrumentation and radiation safety. Chapters 1 through 5 focus on atomic structure and how radiation is produced and interacts with matter. Chapters 6 through 12 walk the reader through the principles and quality assurance of nuclear medicine instruments; $\gamma$-cameras; and SPECT, PET, CT, and MR scanners. The final chapters, 13 through 16, thoroughly cover counting statistics, dosimetry, radiation safety, and regulations.

The initial 5 chapters are quite comprehensive considering how concise they are. Atomic models are briefly reviewed, whereas concepts such as decay and the table of nuclides are given considerably more attention. These chapters contain most of the math in the book, with the exception of the chapter on statistics. The math is not overwhelming, and examples are provided to demonstrate how the equations are to be used. The concepts covered in these chapters lay the foundation for understanding the chapters to follow.

Chapters 6 through 12 detail the workings of an array of nuclear medicine instrumentation. There is a plethora of images of the inner parts of $\gamma$-cameras, CT scanners, and MR scanners-aspects of the instrumentation that technologists rarely have an opportunity to see. In particular, the images associated with collimators in chapter 7 were helpful. As an educator, I have found the concept of collimation often difficult for new students to grasp. In the preface, Lee shares his approach to writing the book as "tell them what you want to tell them, tell them again, and repeat what you told them." This approach is evident in chapter 7 in his description of collimators.

The chapter on MR is the longest. Considering how new the modality is to nuclear medicine technology, the reader will appreciate the comprehensive overview of how MR images are generated. The chapter includes multiple pictorials to illustrate concepts and equipment. Some pictorials are technical whereas others are creative, such as those that use

COPYRIGHT (c) 2016 by the Society of Nuclear Medicine and Molecular Imaging, Inc. the concept of a racetrack to demonstrate precession. The chapter also includes information on how the physics of MR systems may affect hybrid PET/MR instrumentation and how these hybrids can be constructed differently to meet the challenges of marrying these two modalities. Finally, the MR chapter ends with the essentials of MR safety.

The final chapters, 13 through 16, first briefly cover radiation biology to provide a foundation for the following discussion of radiation safety and dosimetry. One can also find a succinct explanation of radiation regulations in chapter 14. A unique aspect of this publication is chapter 16, which describes dose reduction techniques specific to CT and depicts the impact of those techniques on dosimetry.

The illustrations throughout the book creatively depict nuclear medicine technology concepts that can be difficult to explain. As one example, chapter 4 has a unique graphic in which a wooden barrel pouring ale into a mug is used to demonstrate the transient equilibrium of a molybdenum/ technetium generator. Another example is a creative depiction of iterative reconstruction in the SPECT chapter. Iterative reconstruction can be an abstract concept that is difficult to articulate in words alone. The illustration depicting the process step by step provides one of the most concise and logical explanations that I have encountered and complements a succinct description of how the iterative reconstruction algorithm manipulates the image. These are just two examples of how the illustrations are unique, creative, and helpful in explaining nuclear medicine concepts that are proven to work but difficult to visualize.

Because of the intent and scope of the publication, there are some topics it does not cover. As expected, it does not include procedural protocols for specific examinations. General acquisition and processing techniques, such as those for cardiac imaging and SPECT/ PET image reconstruction, are covered, but computers and their applications, such as PACS, printing, and networking are not. Interestingly, there are few references to solid-state detectors. However, a brief explanation of solid-state detectors is included in the portion of the PET/MR chapter on different hybrid systems. Perhaps future editions will add another unique illustration to depict this new and evolving technology.

Basic Science of Nuclear Medicine: The Bare Bone Essentials is a creative compilation of essential take-away points on nuclear physics, instrumentation, and radiation safety. The illustrations demonstrate complex and abstract nuclear medicine processes and concepts in a simple and concise way and alone are reason enough for purchasing this publication. I would recommend it to students who have difficulty finding the prominent points in their more comprehensive textbooks and to seasoned technologists who may need a refresher or to catch up on new technology such as MR. In addition, I believe that having access to this publication in the nuclear medicine department would be most welcome to those inquisitive patients who ask how things work. A picture says a thousand words. The pictures in Basic Science of Nuclear Medicine: The Bare Bone Essentials are worth a thousand (simple-to-understand) words.

\author{
Robert J. Loch, PhD, MBA, CNMT \\ Mercy College of Health Sciences \\ 928 Sixth Ave. \\ Des Moines, IA 50309 \\ E-mail: rloch@mercydesmoines.org
}

Published online Jan. 14, 2016. DOI: 10.2967/jnmt.115.171041 